\title{
BLOOD VESSEL DENSITY AS A PREDICTOR IN ORAL SQUAMOUS CELL CARCINOMA USING CD34
}

\author{
Alaa A. Elmorsy ${ }^{1} B D S$, Taissir A. Omar ${ }^{2} P h D$, Hamed A. Fouad ${ }^{2} P h D$, Mohamed M. Fata ${ }^{3} P h D$
}

\begin{abstract} status. significantly correlated. No significant relation with the histological grade site was found. metastasis of OSCC. But, no correlation was found between the MVD and the histological grading.

KEYWORDS: OSCC, CD34, MVD, angiogenesis, lymph node.

1-Instructor in Oral Pathology Department, Faculty of Dentistry, Alexandria University.

2-Professor in Oral Pathology Department.

3-Professor in Cranio Maxillofacial and Plastic Surgery.

Corresponding author:

E-mail: dr-never-mind@windowslive.com
\end{abstract}

INTRODUCTION: Oral squamous cell carcinoma (OSCC) accounts for more than $90 \%$ of all oral cancers. Despite advances in the approach to locally advanced disease, about $50 \%$ of the tumors will recur. Fifty percent of patients present nodal involvement that is detectable during diagnosis. Of this group, less than $40 \%$ survive after five years, compared with a $90 \%$ survival rate for patients without metastasis. If the lesion is detected at an early stage and treated effectively, the survival rate could exceed 50\%. Many retrospective studies have shown that microvessel density (MVD) could represent a valid independent prognostic factor for the overall survival. The blood vessel density may constitute a relevant parameter for determining the prognosis and guiding biological treatments for aggressive OSCC

OBJECTIVES: To assess the MVD using CD34 in lymph node positive and negative cases and to correlate the results with the lymph node

MATERIALS AND METHODS: MVD was calculated in 22 surgical specimens taken from OSCC patients. Biopsies were taken from the primary tumor of eleven cases proved to have positive lymph node and eleven cases with negative lymph nodes. Immunohistochemical (IHC) staining was performed using a Labeled Strept-Avidin Biotin complex method (LSAB), using the CD34 antibody.

RESULTS: OSCC biopsies showed immunoreactivity to CD34, the difference between the mean MVD and lymph node status was

CONCLUSIONS: MVD was significantly correlated with lymph node status and it could be used as an indicator for progression and early

\section{INTRODUCTION}

Head and neck cancer (HNC) is the sixth most common cancer with 500,000 cases diagnosed per year worldwide (1). Despite advances in the approach to locally advanced disease, about $50 \%$ of patients may relapse (2). Fifty percent of patients present nodal involvement that is detectable at the moment of diagnosis. Of this group, less than $40 \%$ of the patients survive after five years, compared to a $90 \%$ survival rate for patients without metastasis. Therefore, the existence of nodal metastasis decreases the survival rate by $50 \%(3,4)$.

Classic therapeutic modalities of oral squamous cell carcinoma (OSCC) include surgery, radiotherapy, and/or chemotherapy. Although some progress in these treatment methods has occurred, OSCC still shows significant mortality rates (5). If lesions are detected at an early stage and treated effectively, the 5-year survival rate of OSCC patients could exceed 50\%. Therefore, an understanding of the molecular mechanisms involved in OSCC progression would be helpful to attain early detection and develop more effective treatments (6).

Angiogenesis is defined as the growth of new capillaries from preexisting blood vessels. It is observed in wound healing, reproduction, immune reactions and other diseases such as proliferative retinopathies, age-related macular degeneration, and rheumatoid arthritis $(7,8)$.

Within the tumors, development and metastasis require the induction of angiogenesis from a pre-existing vascular network, which guarantees the delivery of oxygen, nutrients and growth factors (9). For this reason, tumors with a higher vessel density seem to show a growth advantage and to progress earlier than the tumors with a poor vascular background (10).

Many retrospective studies have shown that the microvessel density (MVD) could represent a valid independent prognostic factor for the overall survival. Furthermore, a significant correlation between high intratumoral micro vascularization, the presence of metastasis and poor prognosis was detected (11-14).

The anti-CD34 antibody is a monoclonal antibody that recognizes a cell-surface antigen of approximately 110 $\mathrm{kDa}$ that is expressed selectively in human hematopoietic progenitor cells and in the vascular endothelium (15).

It was found that CD34 may enhance the proliferation and block the differentiation of stem or progenitor cells, and promote lymphocyte adhesion to vascular endothelium in lymphoid tissues (16). The exact function of CD34 has not yet been determined (17).

The presence and density of blood vessels in OSCC tissues should play a key role in the progression, dissemination and metastasis of these tumors. However, the evidence is still insufficient (18).

The aim of the present work was to assess the microvessel density in the primary tumor of lymph node positive and negative OSCC cases and to correlate it with the lymph node status.

\section{MATERIALS AND METHODS}

The present study was performed in the Faculty of Dentistry, Alexandria University after gaining the approval 
of the Research Ethics Committee. Twenty-two OSCC patients collected from the Cranio-Maxillofacial and Plastic Surgery Department were included. Biopsies were taken from the primary tumor of eleven cases proved to have positive lymph node and eleven cases with negative lymph nodes. The biopsies were confirmed histopathologically in the Oral Pathology Department at the Faculty of Dentistry, Alexandria University. Patients who presented any signs of active infection, autoimmune diseases, or having a history of radiotherapy, chemotherapy or other cancers were excluded from the study.

The specimens were fixed in $10 \%$ neutral buffered formalin, processed and embedded in paraffin wax using the conventional procedures. Serial sections of 3-4 $\mu \mathrm{m}$ thickness were placed on glass slides and stained using Hematoxylin and Eosin (H\&E). Immunohistochemical (IHC) staining using the CD34 antibody (US Biological, USA) was also performed using the Labeled StreptAvidin Biotin complex method (LSAB) (19). The sections were then examined and the microvessel density was calculated by the image analyzer computer system using Olympus BX41 (Tokyo, Japan).

\section{Statistical analysis}

The difference in the mean microvessel density (MVD) in OSCC cases with positive and negative lymph nodes was estimated using student $(\mathrm{t})$ test.

A (P) value less than 0.05 was considered significant. The values were given as a mean value \pm SD (standard deviation).

\section{RESULTS}

\section{Clinical Results}

The demographic data of the patients included in the current study are shown in Table 1 . In the present study, 22 patients with OSCC were included. The patients' age ranged between 35 and 76 years. Twelve patients (54.54\%) were males and ten patients (45.45\%) were females.

Table 1: Distribution of the studied OSCC cases according to demographic data.

\begin{tabular}{|c|c|c||}
\hline & $(\mathbf{n = 2 2 )}$ & \% \\
\hline Age & \multicolumn{2}{|c|}{} \\
\hline$\leq 60$ & 12 & 54.54 \\
\hline$>60$ & 10 & 45.45 \\
\hline
\end{tabular}

\begin{tabular}{||c|c|c||}
\hline Min. - Max. & \multicolumn{2}{|c|}{$35-76$} \\
\hline Mean \pm SD. & \multicolumn{2}{|c|}{$57.9 \pm 10.9$} \\
\hline Median & \multicolumn{2}{|c|}{59.5} \\
\hline Gender & \multicolumn{2}{|c|}{} \\
\hline Male & 12 & 54.54 \\
\hline Female & 10 & 45.45 \\
\hline Clinical Variants & & \\
Ulcerative & 19 & 86.36 \\
Leukoplakic & 2 & 9 \\
Exophytic & 1 & 4.54 \\
\hline Lymph node metastasis & & 50 \\
Positive & 11 & 50 \\
Negative & 11 & \\
\hline \hline
\end{tabular}

The most common site of occurrence was the lateral side of the tongue $(45.45 \%)$. The second common site of OSCC was the buccal mucosa (27.2\%), followed by the alveolar mucosa (9\%). Finally, the floor of the mouth, and the ventral surface of the tongue, the tip of the tongue and the palate were the least occurring site (4.5\%) for each.

Out of the 22 studied cases, 19 (86.36\%) were ulcerative lesions, while 2 cases (9\%) were presented as white patches. In addition, 1 case (4.54\%) was exophytic forming fungating mass.

\section{Histopathological Results}

The microscopical examination revealed that $82 \%$ of the cases were moderatly differentiated. Each of the well and poorly differentiated squamous cell carcinoma accounted for $9 \%$ of the cases.

\section{Immunohistochemical Results}

All the studied cases (100\%) showed immunoreactivity to CD34 antibody. The endothelial lining of the blood vessels was strongly stained with the antibody CD34 whereas the lymph vessels showed no immunolabeling. (Figure 1 and 2)

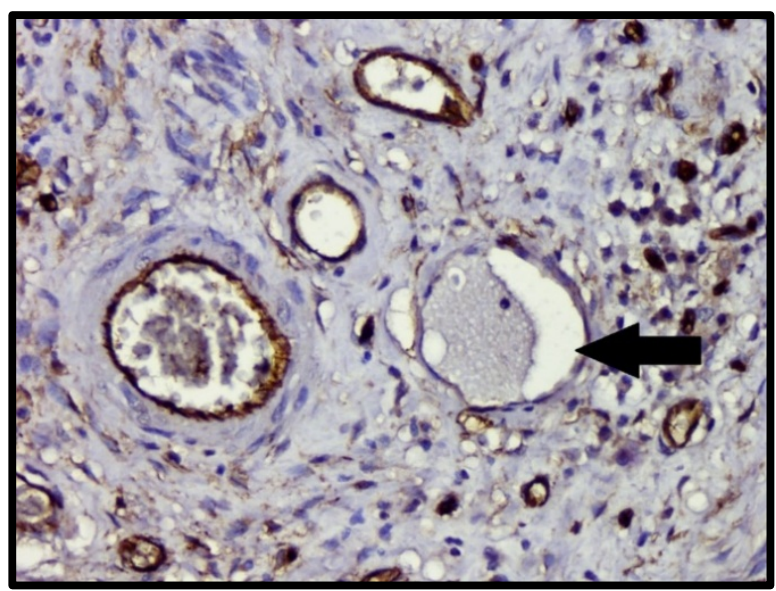

Figure 1: Moderatly differentiated OSCC revealing the specifity of CD34 to blood vessels. notice the negative immunolabling of the neighboring lymph vessel which is filled with lymph(Arrow). (Immuno stain of cd34 x 400). 


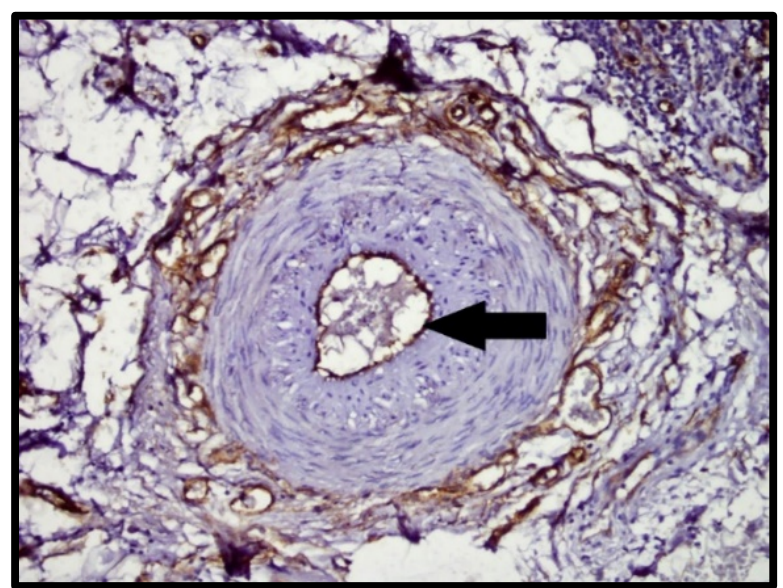

Figure 2: Moderately differentiated OSCC showing large blood vessel surrounded by a large number of smaller ones. notice the specific CD34 staining of the endothelial lining (Arrow). (Immuno stain of cd34 x400).

Collapsed, tortuous, small vessels were observed in the tumor stroma and immersed between the tumor cells (intra-tumoral). In contrast, the vessels located at the edge of the tumors were often enlarged and dilated (peritumoral).

In the present work, it was interesting to observe microscopically the invasion of the malignant epithelial cells into the surrounding structures. This includes the skeletal muscles, nerves, fat cells and blood vessels (Figure 3)

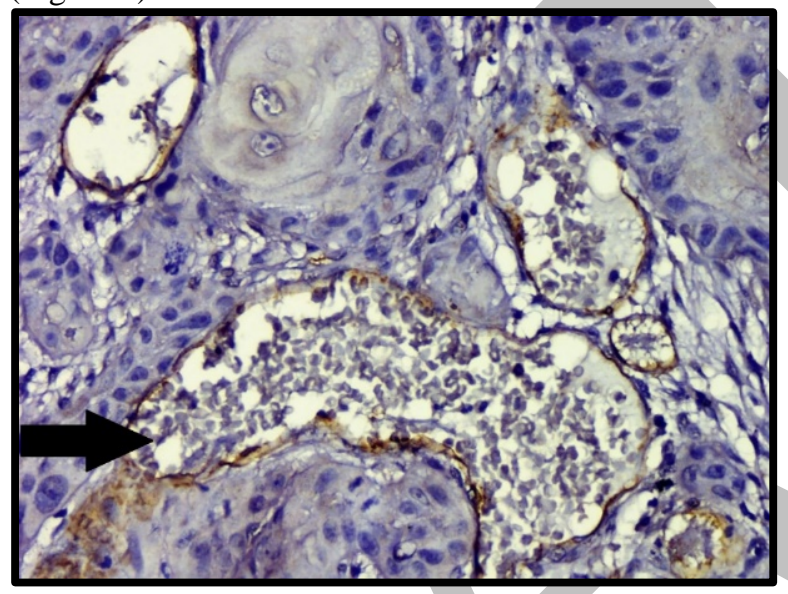

Figure 3: Moderately differentiated OSCC showing epithelial cell nest in close vicinity to a stained blood vessel filled with RBCs (Arrow). (Immuno stain of cd34 x400).

The average number of the blood vessels per $\mathrm{mm} 2$ (MVD) for the lymph node positive OSCC cases was $49.77 \pm 10.66$. while it was $25.27 \pm 3.32$ for the lymph node negative cases.

Regarding the histological grading, the number of vessels in the well differentiated OSCC cases was relatively lesser than the moderately differentiated cases. On the other hand, the poorly differentiated cases had greater number of vessel than the moderately differentiated cases (Figure 4,5 and 6).

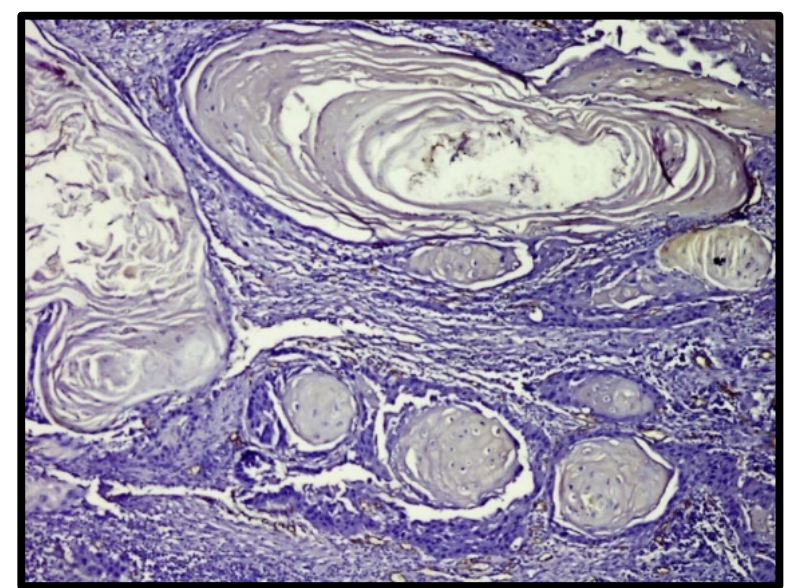

Figure 4: Well differentiated OSCC with negative lymph node showing few number of stained blood vessels in the stroma between the keratin and epithelial pearls (Immuno stain of cd34 x 100).

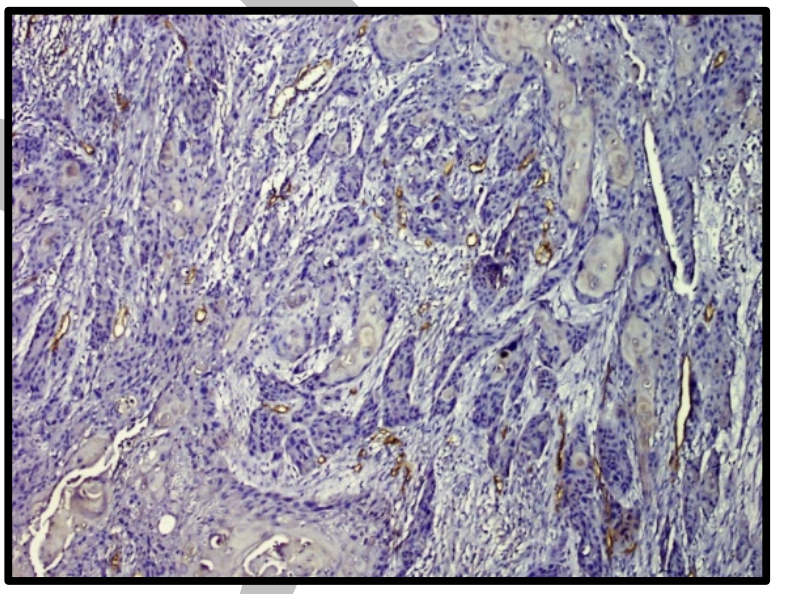

Figure 5: Moderately differentiated OSCC with negative lymph node showing increased number of small irregular stained blood vessels between cell nests (Immuno stain of cd34 x 100).

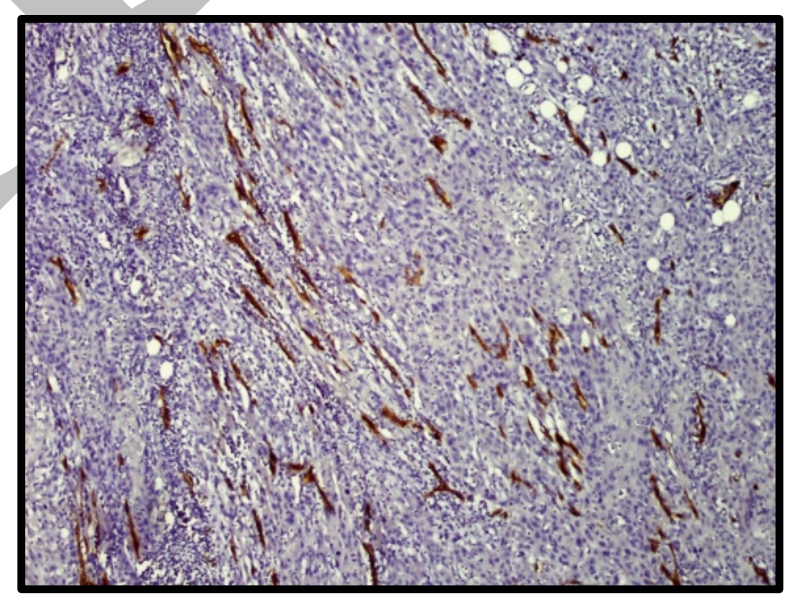

Figure 6: Poorly differentiated OSCC with positive lymph node revealing numerous number of small collapsed tortuous stained blood vessels between the tumor cells (Immuno stain of cd34 x 100).

\section{Microvessel density correlation with the lymph node status of OSCC}

The difference in the mean microvessel density (CD34) between the lymph node positive and negative groups revealed statistical significance at $(\mathrm{P} \leq 0.05)$ using student ttest. (Tables 2)

Table 2: Comparison between the two studied groups with MVD. 


\begin{tabular}{|c|c|c|c|c||}
\hline & \multicolumn{2}{|c|}{ Lymph node status } & \multirow{2}{*||}{ t } & p \\
\cline { 2 - 3 } & $\begin{array}{c}\text { Negative } \\
\text { (n=11) }\end{array}$ & $\begin{array}{c}\text { Positive } \\
\text { (n=11) }\end{array}$ & t & \\
\hline MVD & & & & \\
Min. - Max. & $21.50-33.0$ & $\begin{array}{c}38.50- \\
71.50\end{array}$ & & \\
Mean \pm SD. & $25.27 \pm 3.32$ & $49.77 \pm$ & & \\
& & 10.66 & $7.276^{*}$ & $<0.001^{*}$ \\
Median & 25.0 & 49.0 & & \\
\hline
\end{tabular}

$\mathrm{t}, \mathrm{p}$ : $\mathrm{t}$ and $\mathrm{p}$ values for Student t-test for comparing between the two groups

*: Statistically significant at $\mathrm{p} \leq 0.05$

\section{Microvessel density correlation with the histological grading of OSCC}

There was no significant difference between microvessel density in the well, moderate and poorly differentiated groups and the histological grading of the tumor using ANOVA test (Table 3).

Table 3: Relation between MVD with the histological grading.

\begin{tabular}{|l|c|c|c|c|c||}
\hline \multirow{2}{*}{} & \multicolumn{3}{|c|}{ Grade } & & \\
\cline { 2 - 6 } & $\begin{array}{c}\text { Poor } \\
(\mathbf{n}=2)\end{array}$ & $\begin{array}{c}\text { Moderat } \\
\mathbf{e} \\
(\mathbf{n}=\mathbf{1 8})\end{array}$ & $\begin{array}{c}\text { Well } \\
(\mathbf{n}=\mathbf{2})\end{array}$ & F & P \\
\hline MVD & & & & & \\
Min. - & $33.0-$ & $21.50-$ & $21.50-$ & & \\
Max. & 71.50 & 65.0 & 40.50 & & \\
Mean \pm & $52.25 \pm$ & $36.61 \pm$ & $31.0 \pm$ & 1.263 & 0.305 \\
SD. & 27.22 & 13.49 & 13.44 & & \\
Median & 52.25 & 33.0 & 31.0 & & \\
\hline
\end{tabular}

F,p: $\mathrm{F}$ and $\mathrm{p}$ values for ANOVA test

\section{DISCUSSION}

The proliferation, invasion and metastasis of many cancers are angiogenic dependent; they require neovascularization to grow beyond $1 \mathrm{~mm}$ (20). The degree of neovascularization can be measured by microvessel density. This method was developed by Weidner et al. (13), by using factor VIII that stains mainly mature vessels. Recent antibodies are used now to react not only with normal vessels but also with newly formed vessels trapped within tumour tissues and are thus referred to as pan endothelial markers. CD34 which has been used in the present study, is one of such antibodies $(21,22)$.

It has been found, in different types of human solid tumors that highly vascularized tumors have a significantly higher locoregional LN metastasis than the poorly vascularized tumors (23).

Increased MVD values reportedly are associated with metastasis, advanced tumor stage, and a poor prognosis in many kinds of malignancies (24-26). The first quantitative evidence that angiogenesis in tumors can predict the probability of metastasis has been reported for melanoma (27). However, some reports were unable to find a relation between tumor prognosis and MVD $(28,29)$. On the other hand, another study has found an association with high MVD and better prognosis (30).

The current data showed that MVD was correlated positively with lymph node involvement, which was in accordance with Miyahara et al. (19). This finding implies that high MVD values may predict more aggressive tumor behavior in OSCC and lymph node metastasis. On the other hand, another study found no correlation between the MVD and any clinicopathological parameters (31).

Differences between various studies could be due to different antibodies used (CD 31, CD 34 and factor VIII) by authors to define endothelium and different methodologies used in assessment of various parameters besides interobserver variation. This was in agreement with Astekar et al. (32), who also mentioned that the differences between immunohistochemical protocols, like selection of the paraffin block, level of section within the tissue block, that is, superficial or deep and variability in the selection of hot spot identification may contribute to the variation of the results amongst different researchers. In addition, the lack of a standardized direct method to measure angiogenesis as a factor was reported by Tae et al (33).

In the present study, it was found that no significant associations were observed between MVD and the histologic grade. This finding was consistent with some authors $(34,35) \quad(\mathrm{P}>0.05)$. they also confirmed that carcinomas present substantial heterogeneity in their vascularity.

On the other hand, in some studies the microvessel density was observed to be higher when OSSC was less differentiated $(36,37)$. This could be attributed to the differences in patient selection, methodology, and the different anatomic sites included in the analysis (38).

The results of the current study support the possibility that MVD could be used as a prognostic factor. Further studies using angiogenesis inhibitors may form an important component of therapeutic strategies aiming invasive metastatic OSCC. Secondly, it may become possible to interfere with initial tumor development by blocking the angiogenic switch that precedes the progression to invasive cancer (39).

\section{CONCLUSION}

MVD was significantly correlated with lymph node status and it could be used as an indicator for progression and early metastasis of OSCC. While, no correlation was found between the MVD and the histological grading.

\section{CONFLICT OF INTEREST}

The authors declare that they have no conflicts of interest.

\section{REFERENCES}

1. Siegel R, Ma J, Zou Z, Jemal A Cancer statistics, 2014. CA Cancer J Clin .2014;64:9-29.

2. Vermorken J, Mesia R, Rivera F, Remenar E, Kawecki A, et al. Platinum-based chemotherapy plus cetuximab in head and neck cancer. N Engl J Med.2008; 359: 1116-27.

3. Sano D, Myers JN. Metastasis of squamous cell carcinoma of the oral tongue. Cancer Metastasis Rev 2007; 26: 64562.

4. Grimm M. Prognostic value of clinicopathological parameters and outcome in 484 patients with oral squamous cell carcinoma: Microvascular invasion $(\mathrm{V}+)$ is an independent prognostic factor for OSCC. Clin Transl Oncol 2012; 14: 870-80.

5. Pianka A, Knosel T, Probst F, Troeltzsch M, Woodlock T, Otto S, et al. Vascular endothelial growth factor receptor isoforms: are they present in oral squamous cell 
carcinoma? Journal of Oral and Maxillofacial Surgery. 2015;73:897-904.

6. Liping S, Ying W, Mingzhen X, Yuan L, Lei Y. Upregulation of survivin in oral squamous cell carcinoma correlates with poor prognosis and chemoresistance. Oral Surgery, Oral Medicine, Oral Pathology, Oral Radiology, and Endodontology. 2010;110:484-91.

7. Folkman J, Haudenschild C. Angiogenesis in vitro. Nature. 1980; 288:551-6.

8. Folkman J. Angiogenesis in cancer, vascular, rheumatoid and other disease. Nat Med. 1995; 1:27-31.

9. Folkman J. How is blood vessel growth regulated in normal and neoplastic tissue? G.H.A. Clowes memorial Award lecture. Cancer Res 1986; 46: 467-73.

10. Folkman J. What is the evidence that tumors are angiogenesis dependent? J Natl Cancer Inst.1990; 82:4-6.

11. Weinder N. Tumor vascularity as a prognostic factor in cancer patients: The evidence continues to grow. J Pathol 1998; 184:119-22.

12. Macchinarini P, Fontanini G, Hardin MJ, Squatini F, Angeletti CA. Relation of neovascularization to metastasis of non-small cell lung cancer. Lancet 1992; 340:145-6.

13. Weinder N, Semple JP, Welch WR, Folkman J. Tumor angiogenesis and metastasis correlation in invasive breast carcinoma. N Engl J Med 1991; 324:1-8.

14. Brawer MK, Deering RE, Brown M, Preston SD, Bigler SA. Predictors of pathologic stage in prostatic carcinoma cancer 1994; 73:678-87.

15. Fina L, Molgaard HV, Robertson D, Bradley NJ, Monaghan P, Delia D, et al. Expression of the CD34 gene in vascular endothelial cells. Blood 1990; 75: 2417-26.

16. Nielsen JS, McNagny KM. Novel functions of the CD34 family. Journal of Cell Science. 2008;121:3683-92.

17. Ettl T, Hautmann M, Reichert TE, Bauer R. Cycling CD34 expression in subpopulations of head and neck squamous cell carcinoma cell lines is involved in radioresistance and change in cytokeratin expression profile. Clinical and experimental medicine. 2017;17:565-74.

18. Mashhadiabbas F, Mahjour F, Mahjour SB, Fereidooni F, Hosseini FS. The immunohistochemical characterization of MMP-2, MMP-10, TIMP-1, TIMP-2, and podoplanin in oral squamous cell carcinoma. Oral Surg Oral Med Oral Pathol Oral Radiol 2012; 114: 240-50.

19. Miyahara M, Tanuma Ji, Sugihara K, Semba I. Tumor lymphangiogenesis correlates with lymph node metastasis and clinicopathologic parameters in oral squamous cell carcinoma. Cancer. 2007;110:1287-94.

20. Bryne M, Koppang HS, Lilleng R, Stene T, Bang G, Dabelsteen E. New malignancy grading is a better prognostic indicator than Broders' grading in oral squamous cell carcinomas. J Oral Pathol Med. 1989;18:432-7.

21. Shieh YS, Lee HS, Shiah SG, Chu YW, Wu CW, Chang LC. Role of angiogenic and non angiogenic mechanisms in oral squamous cell carcinoma: correlation with histologic differentiation and tumor progression. Journal of Oral Pathology \& Medicine. 2004;33:601-6.

22. Safwat M, Habib F, Elayat A, Oweiss N, Reffat S, Algaidi S. Morphometric and immunohistochemical study of angiogenic marker expressions in invasive ductal carcinomas of the human breast. Folia Morphologica. 2009;68:144-55.
23. Gasparini G. Prognostic and predictive value of intratumoral microvessel density in human solid tumours. Tumour Angiogenesis. 1997:29-44.

24. Chien CY, Su CY, Hwang CF, Chuang HC, Hsiao YC, $\mathrm{Wu} \mathrm{SL}$, et al. Clinicopathologic significance of CD105 expression in squamous cell carcinoma of the hypopharynx. Head \& Neck. 2006;28:441-6.

25. Dales J-P, Garcia S, Bonnier P, Duffaud F, Andrac-Meyer L, Ramuz O, et al. CD105 expression is a marker of high metastatic risk and poor outcome in breast carcinomas: correlations between immunohistochemical analysis and long-term follow-up in a series of 929 patients. American Journal of Clinical Pathology. 2003;119:374-80.

26. Wikström P, Lissbrant IF, Stattin P, Egevad L, Bergh A. Endoglin (CD105) is expressed on immature blood vessels and is a marker for survival in prostate cancer. The Prostate. 2002;51:268-75.

27. Srivastava A, Laidler P, Davies R, Horgan K, Hughes L. The prognostic significance of tumor vascularity in intermediate-thickness $\quad(0.76-4.0 \quad \mathrm{~mm}$ thick $)$ skin melanoma. A quantitative histologic study. The American Journal of Pathology. 1988;133:419.

28. Fox SB, Gatter KC, Harris AL. Tumour angiogenesis. The Journal of Pathology. 1996;179:232-7.

29. Page DL, Jensen RA. Angiogenesis in human breast carcinoma: what is the question?: WB Saunders; 1995.

30. Lindmark G, Gerdin B, Sundberg C, Påhlman L, Bergström R, Glimelius B. Prognostic significance of the microvascular count in colorectal cancer. Journal of Clinical Oncology. 1996;14:461-6.

31. de Carvalho Fraga CA, de Oliveira MVM, de Oliveira ES, Barros LO, Domingos PLB, Gomes ÉPP, et al. prognostic value of intratumoral cd57+ t cells and microvessel density in head and neck squamous cell carcinoma. Archives of Oral Research. 2017;6.

32. Astekar M, Joshi A, Ramesh G, Metgud R. Expression of vascular endothelial growth factor and microvessel density in oral tumorigenesis. Journal of Oral and Maxillofacial Pathology: JOMFP. 2012;16:22.

33. Tae K, El-Naggar AK, Yoo E, Feng L, Lee JJ, Hong WK, et al. Expression of vascular endothelial growth factor and microvessel density in head and neck tumorigenesis. Clinical Cancer Research. 2000;6:2821-8.

34. de Sousa SF, Gleber-Netto FO, de Oliveira-Neto HH, Batista AC, Abreu MHNG, de Aguiar MCF. Lymphangiogenesis and podoplanin expression in oral squamous cell carcinoma and the associated lymph nodes. Applied Immunohistochemistry \& Molecular Morphology. 2012;20:588-94.

35. Riedel F, Götte K, Bergler W, Rojas W, Hörmann K. Expression of basic fibroblast growth factor protein and its down regulation by interferons in head and neck cancer. Head \& Neck. 2000;22:183-9.

36. Tan ZZ, Hossain MZ, Ahsan MS, Chew YC. Microvessel density in different grades of oral squamous cell carcinoma and its relationship with keratin pearl. Sains Malaysiana. 2016;45:201-5.

37. Xia X, Du R, Zhao L, Sun W, Wang X. Expression of AEG-1 and microvessel density correlates with metastasis and prognosis of oral squamous cell carcinoma. Human Pathology. 2014;45:858-65.

38. Zhao D, Pan J, Li XQ, Wang XY, Tang C, Xuan M. Intratumoral lymphangiogenesis in oral squamous cell 
carcinoma and its clinicopathological significance. J Oral Pathol Med. 2008;37:616-25.

39. Hanahan D, Folkman J. Patterns and emerging mechanisms of the angiogenic switch during tumorigenesis. Cell. 1996;86:353-64.

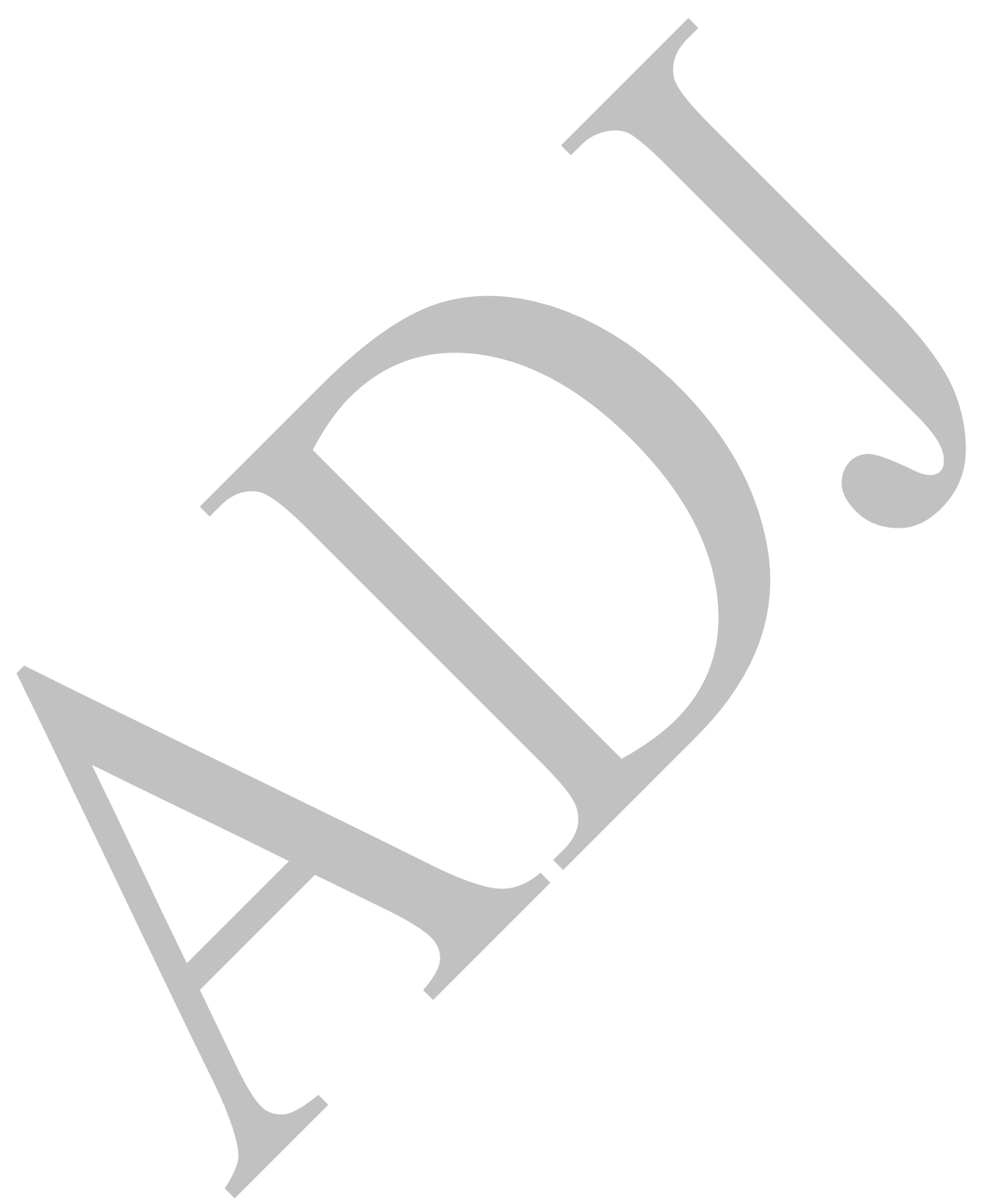

